\title{
Effects of Modafinil on Behavioral Learning and Hippocampal Synaptic Transmission in Rats
}

\author{
Wen-Wen Yan ${ }^{1, *}$, Li-Hua Yao ${ }^{2, *}$, Chong Chen ${ }^{2}$, Hai-Xia Wang ${ }^{3}$, Chu-Hua Li ${ }^{1}$, Jun-Ni Huang ${ }^{1}$, Peng Xiao ${ }^{1}$, Cheng-Yi Liu ${ }^{3}$ \\ ${ }^{1}$ School of Life Science, South China Normal University, Guangzhou, China \\ ${ }^{2}$ School of Life Science, Jiangxi Science \& Technology Normal University, Nanchang, China \\ ${ }^{3}$ School of Sport Science, South China Normal University, Guangzhou, China
}

Purpose: Modafinil is a wake-promoting agent that has been proposed to improve cognitive performance at the preclinical and clinical levels. Since there is insufficient evidence for modafinil to be regarded as a cognitive enhancer, the aim of this study was to investigate the effects of chronic modafinil administration on behavioral learning in healthy adult rats.

Methods: Y-maze training was used to assess learning performance, and the whole-cell patch clamp technique was used to assess synaptic transmission in pyramidal neurons of the hippocampal CA1 region of rats.

Results: Intraperitoneal administration of modafinil at $200 \mathrm{mg} / \mathrm{kg}$ or $300 \mathrm{mg} / \mathrm{kg}$ significantly improved learning performance. Furthermore, perfusion with $1 \mathrm{mM}$ modafinil enhanced the frequency and amplitude of spontaneous postsynaptic currents and spontaneous excitatory postsynaptic currents in CA1 pyramidal neurons in hippocampal slices. However, the frequency and amplitude of spontaneous inhibitory postsynaptic currents in CA1 pyramidal neurons were inhibited by treatment with $1 \mathrm{mM}$ modafinil.

Conclusions: These results indicate that modafinil improves learning and memory in rats possibly by enhancing glutamatergic excitatory synaptic transmission and inhibiting GABAergic (gamma-aminobutyric acid-ergic) inhibitory synaptic transmission.

Keywords: Modafinil; Learning; CA1 Region, Hippocampal; Synaptic Transmission; Synaptic Potentials

- Grant Support: This study was supported by the National Natural Science Foundation of China (81360205) to Li-Hua Yao.

- Research Ethics: The protocol for the care and use of animals and the experimental protocol of this study were approved by the Institutional

Care and Use Committee of South China Normal University

- Conflict of Interest: No potential conflict of interest relevant to this article was reported.

\section{INTRODUCTION}

Modafinil, (diphenyl-methyl)-sulfinyl-2-acetamide, is a wellknown wake-promoting drug (Fig. 1) that is used for the treatment of narcolepsy [1], idiopathic hypersomnia [1], hypoglyce- mia [2], multiple sclerosis [3], and Parkinson disease [4]. In addition to treating excessive daytime sleepiness, modafinil has been shown to enhance attention and working memory in sleep-deprived humans and rodents [5,6]. Because of the wide off-label use of modafinil in healthy, non-sleep-deprived indi-

Corresponding author: Cheng-Yi Liu (iD http://orcid.org/0000-0003-3697-6534 School of Sport Science, South China Normal University, Guangzhou, Guangdong, 51006, China

E-mail: 452698560@qq.com / Tel: +86-20-85217713 / Fax: +86-79183815794

-Wen-Wen Yan (iD http://orcid.org/0000-0002-7499-9852

- Li-Hua Yao (iD http://orcid.org/0000-0002-4112-1920

*Wen-Wen Yan and Li-Hua Yao contributed equally to this study as co-first authors.

Submitted: August 11, 2015 / Accepted after revision: September 16, 2015 


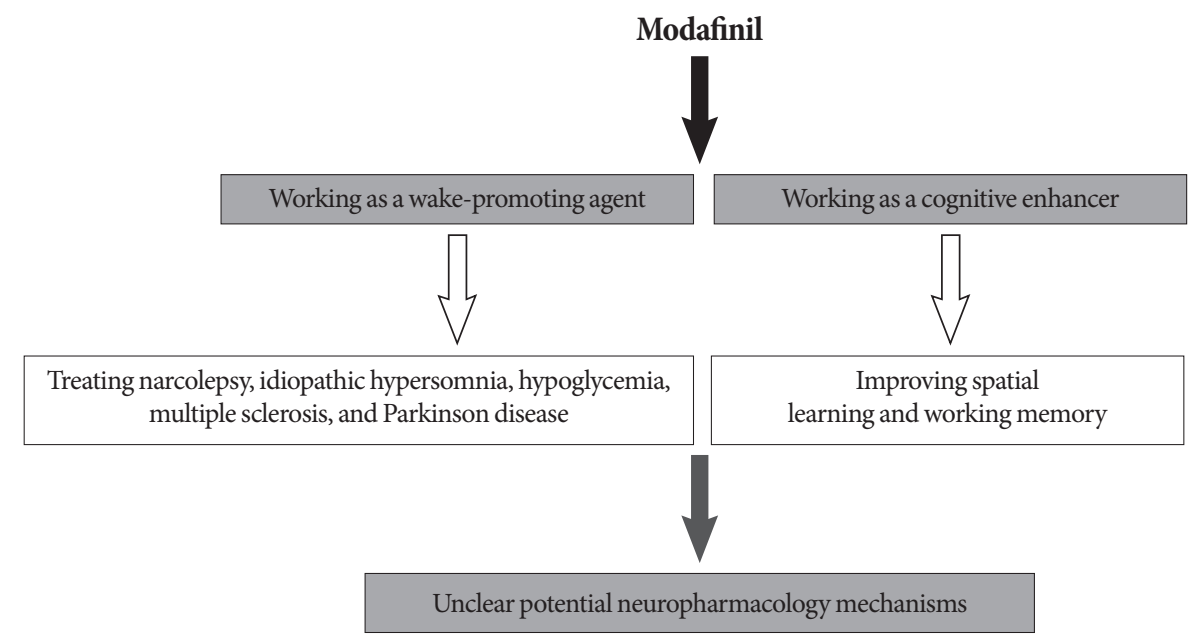

Fig. 1. A schematic diagram illustrating the positive effects of modafinil as a wake-promoting agent and cognitive enhancer.

viduals, the effects of modafinil on cognition have been investigated at the preclinical and clinical level. Modafinil has been reported to enhance performance on the digit-span task, visual recognition memory tests, spatial planning, and symbol substitution reaction times in healthy, non-sleep-deprived adults, suggesting improved working memory (Fig. 1) [5]. A few studies have focused on the efficacy of modafinil for improving cognitive performance in animal models. It has been reported that treatment with modafinil enhanced performance in a sequential alternation task [7] and improved the rate of spontaneous alternation behavior in mice [8], indicating that modafinil may enhance working memory. With regard to the effects of modafinil on long-term memory, it has been shown that daily modafinil administration before training improved learning on a serial spatial discrimination reversal task [9], and acquisition on the Morris water maze [10]. Chronic (repeated) modafinil administration has also resulted in a favorable performance profile for visuospatial tasks (typically, a hippocampus-dependent task) but markedly decreased successful responses in complex operant conditioning (typically, a prefrontal cortex-dependent task) [11]. In addition, modafinil has been observed to have no effect on cued fear memory that is hippocampus-independent [10]. Thus, there is still insufficient evidence for modafinil to be considered a cognitive enhancer.

Synaptic transmission, the essential process in brain physiology and pathology, is critical for signal integration by the central nervous system, especially in learning and memory. Learning performance may be affected by changes in either excitatory or inhibitory synaptic transmission. The enhancement of excit- atory transmission in the hippocampal dentate gyrus contributes to the facilitation of Y-maze learning performance [12], while enhanced inhibitory synaptic transmission in the dentate gyrus is found in animals that exhibit impaired cognition in behavioral tasks mediated by the hippocampus [13]. Previous studies have shown that modafinil could reduce the release of extracellular $\gamma$-aminobutyric acid (GABA) [6] and increase glutamate release in the hippocampus and thalamus [8], suggesting that it may modulate both excitatory and inhibitory synaptic transmission in the hippocampus.

To further explore the efficacy of modafinil as a cognitive enhancer for learning and memory, the present study investigated the effects of chronic intraperitoneal (i.p.) modafinil administration on Y-maze learning in healthy, non-sleep-deprived adult rats. Furthermore, to obtain information regarding the effects of modafinil on the neural substrates of cognition, the effects of modafinil on spontaneous postsynaptic currents (sP$\mathrm{SCs}$ ), spontaneous excitatory postsynaptic currents (sEPSCs), and spontaneous inhibitory postsynaptic currents (sIPSCs) in the CA1 region of rat hippocampal brain slices were examined in vitro using the whole-cell patch clamp technique.

\section{MATERIALS AND METHODS}

\section{Chemicals and Drugs}

Modafinil, the chemicals used for making artificial cerebrospinal fluid (ACSF), bicuculline, Mg-ATP, Na-GTP, KF, 6-cyano7-nitroquinoxaline-2,3-dione (CNQX), and DL-2-Amino5-phosphonovaleric acid (APV), were purchased from Sigma 
Co. (St. Louis, MO, USA). For the whole-cell patch clamp experiments, modafinil was dissolved in ACSF and its effects were tested by bath perfusion (solution exchange was completed in about 30 seconds).

\section{Animals and Groups}

The protocol for the care and use of animals and the experimental protocol of this study were approved by the Institutional Care and Use Committee of South China Normal University. Healthy, male Sprague-Dawley rats were housed at $22^{\circ} \mathrm{C} \pm 3^{\circ} \mathrm{C}$ and $55 \% \pm 5 \%$ humidity, and maintained on a 12 -hour light $/ 12$ hour dark cycle (lights on at $8 \mathrm{AM}$ ), with free access to food and water.

The effects of different doses of modafinil on the behavioral learning performance of rats were investigated first. Healthy male Sprague-Dawley rats (2-3 months old) were randomly divided into four groups: control, 100, 200, and $300 \mathrm{mg} / \mathrm{kg}$ modafinil groups. Modafinil groups were treated with modafinil dissolved in saline, while the control group was treated with saline. In each daily trial, 1-1.5 hours before behavioral learning tests, the rats received a dose of 100, 200, or $300 \mathrm{mg} / \mathrm{kg}$ i.p. modafinil (M100, $100 \mathrm{mg} / \mathrm{kg} ;$ M200, $200 \mathrm{mg} / \mathrm{kg} ;$ M300, $300 \mathrm{mg} / \mathrm{kg}, \mathrm{n}=8$ rats for each group) or saline alone ( $\mathrm{n}=8$ rats). Daily administration was continued until the end of the Y-maze task. All modafinil or saline injections and behavioral tests were performed between $11 \mathrm{AM}$ and $5 \mathrm{PM}$.

\section{Behavioral Learning Performance}

Behavioral learning was assessed using a Y-maze (MG-3S, Zhenghua Machine Co., Anhui, China) with three identical radial arms. During the behavioral training, the Y-maze was located in a darkened room. The animals were placed at the intersection of the three arms and were trained to enter a randomly selected, brightly lit arm within the first 10 seconds. A 15-W lamp was suspended at the end of each arm. The choice of a darkened arm was counted as a "discrimination error." Whenever the animal made an error, it received a brief electric footshock (35 V AC, $4.5 \mathrm{~mA}$; duration 1 second, every 5 seconds) until it entered the brightly lit arm. The training procedure was performed on 8 consecutive days and the rats underwent 20 trials at 20-40 seconds random intervals once every 24 hours. An accuracy rate of $90 \%$ served as the learning criterion.

\section{Preparation of Hippocampal Brain Slices}

All experiments were performed on CA1 pyramidal neurons in hippocampal brain slices of rats, as described previously [14,15]. Briefly, animals were anesthetized with isoflurane and decapitated. The brains were quickly removed from the cranial cavity and placed in ice-cold $\left(4^{\circ} \mathrm{C}\right)$ oxygenated ACSF containing (mM): $\mathrm{NaCl} 117, \mathrm{KCl} 4.7, \mathrm{MgCl}_{2} 1.2, \mathrm{NaH}_{2} \mathrm{PO}_{4} 1.2, \mathrm{NaHCO}_{3}$ $25, \mathrm{CaCl}_{2} 2.5$, D-glucose 10, pH 7.4 (after the solution was saturated with $95 \% \mathrm{O}_{2} / 5 \% \mathrm{CO}_{2}$ ). The hippocampus was freely dissected, and $400-\mu \mathrm{m}$ thick transverse hippocampal slices were cut using a tissue chopper (McIllwain, Campden, UK). Slices were then incubated in oxygenated ACSF and maintained at $31^{\circ} \mathrm{C} \pm 0.5^{\circ} \mathrm{C}$. After 90 minutes of incubation, individual slices were transferred to a recording chamber and continuously perfused with ACSF at a rate of $4 \mathrm{~mL} / \mathrm{min}$. All experiments were performed at room temperature $\left(22^{\circ} \mathrm{C}-25^{\circ} \mathrm{C}\right)$.

\section{Whole-Cell Current Recording}

Whole-cell recording was performed in neurons of the CA1 region in rat hippocampal slices to record sPSCs, sEPSCs, and sIPSCs. The sPSCs were recorded using a modification of methods described previously [16]. The membrane potential was held at $-80 \mathrm{mV}$ with pipettes containing $(\mathrm{mM}): \mathrm{CsCl}_{2} 140$, $\mathrm{MgCl}_{2}$ 6, 4-(2-hydroxyethyl)-1-piperazineethanesulfonic acid (HEPES) 10, ethylene glycol tetraacetic acid (EGTA) 10, MgATP 2, Na-GTP 0.1, pH 7.4. Under this experimental condition, both glutamatergic sEPSCs and gamma-aminobutyric acid-ergic (GABAergic) sIPSCs are inward currents [16]. The sEPSCs were recorded in the presence of the GABAa receptor antagonist bicuculline $(20 \mu \mathrm{M})$ at a holding potential of $-70 \mathrm{mV}$ with pipettes containing (mM): $\mathrm{K}$ gluconate $140, \mathrm{MgCl}_{2} 2$, HEPES 10, EGTA 5, Na-ATP 2, Na-GTP 0.2, pH 7.4. Under this experimental condition, the glutamatergic sEPSCs are inward currents. The sIPSCs were recorded in the presence of the AMPA (a-amino-3-hydroxy-5-methyl-4-isoxazole-propionic acid) receptor antagonist CNQX $(20 \mu \mathrm{M})$, and the NMDA (N-methylD-aspartic acid) receptor antagonist APV $(50 \mu \mathrm{M})$, at a holding potential of $-10 \mathrm{mV}$ with pipettes containing $(\mathrm{mM})$ : KF 140 , $\mathrm{NaCl}$ 6, HEPES 10, EGTA 5, Mg-ATP 2, Na-GTP 0.1, pH 7.4. Under this experimental condition, GABAergic sIPSCs are outward currents. The recorded neuronal cells were allowed to stabilize for 5-10 minutes and the effects of modafinil were tested by bath perfusion for 3 minutes.

Data were acquired using Clampex 9.2 (Molecular Devices, Sunnyvale, CA, USA) via a Digidata 1322 series A/D board set to a sampling frequency of $10 \mathrm{kHz}$. After whole-cell access was achieved, the series resistance was partially compensated by the 
amplifier. Both input resistance and series resistance were monitored throughout the experiments. Only those recordings with a stable series resistance $(\leq 20 \mathrm{M} \Omega)$ and input resistance were accepted.

\section{Statistical Analysis}

Synaptic events for sPSCs, sEPSCs, and sIPSCs were counted and analyzed off-line using Mini Analysis software (ver. 6.0.3, Synaptosoft, Decatur, GA, USA). All results were presented as mean \pm standard error of the mean. Statistically significant differences were calculated using Student paired t-test, or the Kolmogorov-Smirnov test. A level of $\mathrm{P}<0.05$ was considered statistically significant.

\section{RESULTS}

\section{Y-maze Learning Performance}

As shown in Fig. 2A, there were no differences in the percentage of correct choices on day 1 for all the four groups $(\mathrm{P}>0.05)$. Although the accuracy rates for the M100 group from days 2 to 8 were a little higher than those of the control group, there were no significant differences $(\mathrm{P}>0.05)$. However, the accuracy rates for the M200 and M300 groups were significantly higher than that for the control group from days 2 to 5 ( $\mathrm{P}<0.01$ and $\mathrm{P}<0.05$, respectively). Furthermore, there were no obvious differences between the M200 and M300 groups in the accuracy rates from days 1 to $8(\mathrm{P}>0.05)$ (Fig. $2 \mathrm{~A})$. The total number of trials to reach the learning criterion in the saline group was $94.63 \pm 14.88$ after 6 days of training (Fig. 2B). The M100,

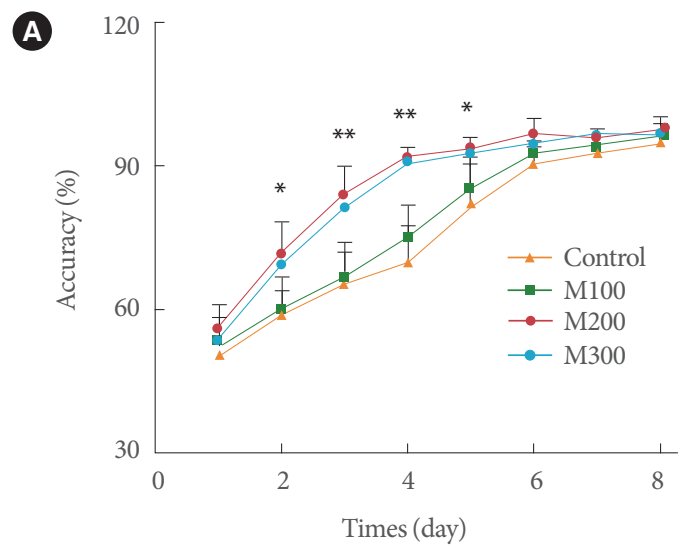

M200, and M300 modafinil groups reached the learning criterion after 6,4 , and 4 days of training, and the total numbers of trials were $91.50 \pm 8.47,59.25 \pm 12.70$, and $58.13 \pm 8.61$, respectively (Fig. 2). These results reveal that the improvement provided by modafinil in Y-maze learning performance was dosedependent and did not increase further when the modafinil concentration was more than $200 \mathrm{mg} / \mathrm{kg}$. Thus, the results suggest that chronic modafinil administration significantly enhanced the cognitive function of the rats.

\section{Impact of Modafinil on sPSCs}

The pyramidal neurons recorded in the CA1 region of the hippocampus exhibited sPSCs when the membrane potential was held at $-80 \mathrm{mV}$. Under those conditions, both glutamatergic sEPSCs and GABAergic sIPSCs are inward currents and the sPSCs are the sum of the sEPSCs and sIPSCs [16]. The effect of different concentrations of modafinil on sPSCs was investigated in rat hippocampal slices. Modafinil increased the frequency and amplitude of sPSCs (Fig. 3A). As shown in Fig. 3B, the frequency of sPSCs as a percentage of the control values increased to $110.22 \% \pm 12.26 \%$ ( 9 slices, 5 animals, $\mathrm{P}>0.05$ ), 187.19\% $\pm 33.79 \%$ ( 9 slices, 5 animals; $\mathrm{P}<0.01$ ), and $202.61 \% \pm 39.34 \%$ (9 slices, 5 animals; $\mathrm{P}<0.01$ ) in response to $0.5,1$, and $2 \mathrm{mM}$ modafinil perfusion, respectively. However, there was no significant difference between the enhancement provided by 1 and $2 \mathrm{mM}$ modafinil treatment $(\mathrm{P}>0.05)$, and the enhancement by $1 \mathrm{mM}$ modafinil diminished quickly after washout (Fig. 3A, B). Thus, a concentration of $1 \mathrm{mM}$ was adopted for the subsequent tests. Furthermore, the cumulative probability curves for the amplitude of sPSCs events record-

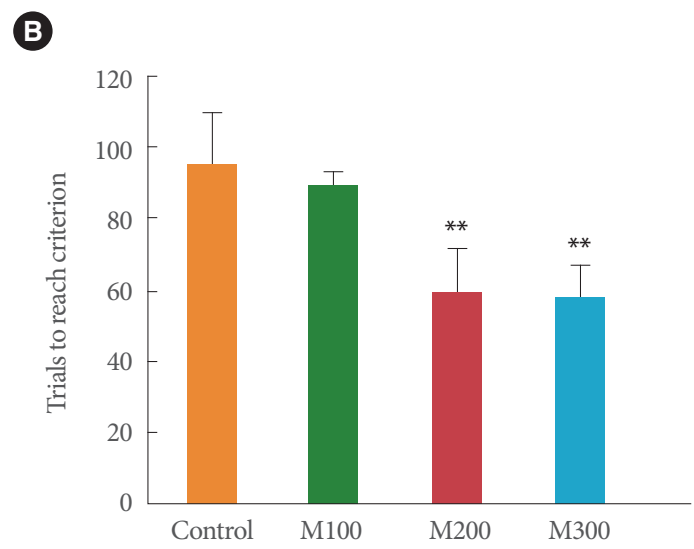

Fig. 2. Effect of modafinil treatment on the Y-maze learning task. (A) Effect of modafinil at concentrations of 100, 200, and $300 \mathrm{mg} / \mathrm{kg}$ on the accuracy rates for the Y-maze learning task $(\mathrm{n}=8)$. (B) Effects of modafinil at concentrations of 100,200 , and $300 \mathrm{mg} / \mathrm{kg}$ on the number of trials required to achieve the learning criterion in the Y-maze task $(\mathrm{n}=8) .{ }^{*} \mathrm{P}<0.05$ and ${ }^{* *} \mathrm{P}<0.01$ compared with the control group. M100, modafinil 100 mg/kg; M200, modafinil 200 mg/kg; M300, modafinil $300 \mathrm{mg} / \mathrm{kg}$. 
A

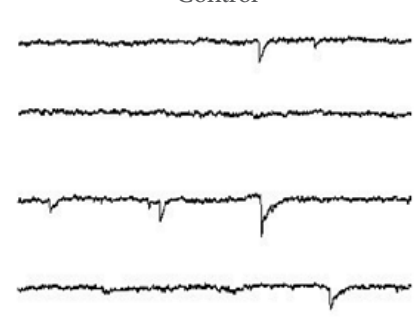

B

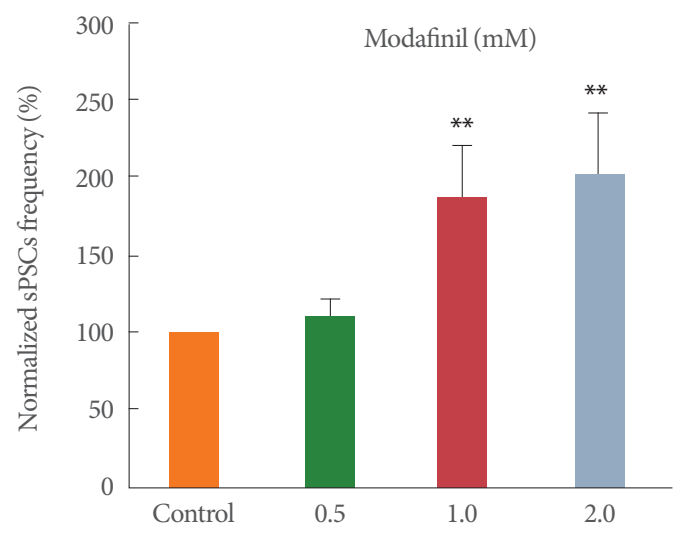

Modafinil

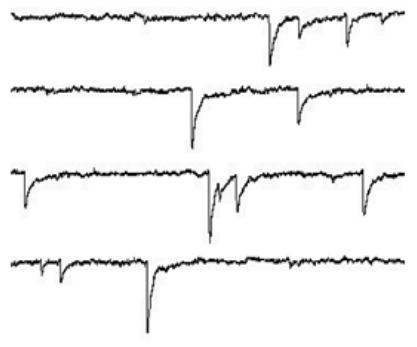

Washout

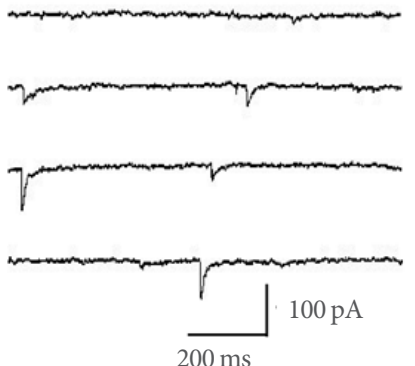

C

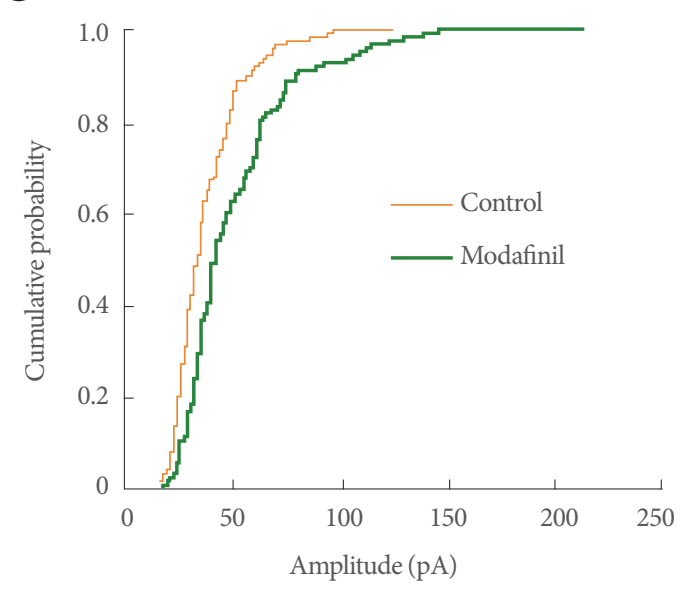

Fig. 3. Modafinil increased the frequency and amplitude of spontaneous postsynaptic currents (sPSCs) in CA1 pyramidal neurons in hippocampal slices. (A) Sample traces represent sPSCs recorded in a CA1 pyramidal neuron during 1mM modafinil treatment. (B) Modafinil at $1 \mathrm{mM}$ or $2 \mathrm{mM}$ concentration increased the frequency of sPSCs $(n=9, P<0.01)$. (C) Cumulative amplitude distribution of sPSCs shows that $1 \mathrm{mM}$ modafinil increased the amplitude of sPSCs ( $\mathrm{n}=9$, Kolmogorov-Smirnov test, $\mathrm{P}<0.01)$. Membrane holding potential, $-80 \mathrm{mV}$. ${ }^{*} \mathrm{P}<0.01$ compared with the control group.

ed in the control and $1 \mathrm{mM}$ modafinil-treated slices were plotted (Fig. 3C), and these showed that the amplitude was also significantly increased by modafinil ( 9 slices, 5 animals, KolmogorovSmirnov test, $\mathrm{P}<0.01)$.

\section{Impact of Modafinil on sEPSCs}

The sEPSCs were isolated by bath application of $20 \mu \mathrm{M}$ bicuculline to block GABAa receptor-mediated synaptic currents on the CA1 pyramidal neurons (Fig. 4A). The sEPSCs were totally blocked by bath coapplication of $20 \mu \mathrm{M}$ CNQX and $50 \mu \mathrm{M}$ APV, confirming that they were glutamate receptor-mediated events (data not shown). The effects of modafinil on sEPSCs were investigated in rat hippocampal slices. As shown in Fig. 4B, the frequency of sEPSCs increased reversibly to $175.14 \% \pm 6.10 \%$ of that of control (9 slices, 5 animals; $\mathrm{P}<0.01$ ) during $1 \mathrm{mM}$ modafinil perfusion. The amplitude of sEPSCs was also significantly increased by modafinil ( 9 slices, 5 animals, Kolmogorov-Smirnov test, $\mathrm{P}<0.01$ ) (Fig. 4C).

\section{Impact of Modafinil on sIPSCs}

The sIPSCs in hippocampal CA1 pyramidal neurons were analyzed at a holding potential of $-10 \mathrm{mV}$, and were isolated pharmacologically from spontaneous excitatory currents by the inclusion of $20 \mu \mathrm{M}$ CNQX and $50 \mu \mathrm{M}$ APV in the ACSF perfusing the slices (Fig. 5A). The sIPSCs were totally blocked by bath application of $20 \mu \mathrm{M}$ bicuculline, confirming that they were GABAa receptor-mediated events (data not shown). The effect of modafinil on sIPSCs was examined and the frequency of sIPSCs was reversibly decreased to $31.12 \% \pm 8.21 \%$ of control by $1 \mathrm{mM}$ modafinil treatment (8 slices, 5 animals; $\mathrm{P}<0.01$ ) (Fig. $5 \mathrm{~A}, \mathrm{~B})$. The cumulative probability curves for the amplitude of sIPSCs events recorded in the control and modafinil-treated slices were plotted, and these showed that the amplitude was also significantly decreased by modafinil $(52.36 \% \pm 18.75 \%$ of 
A

B

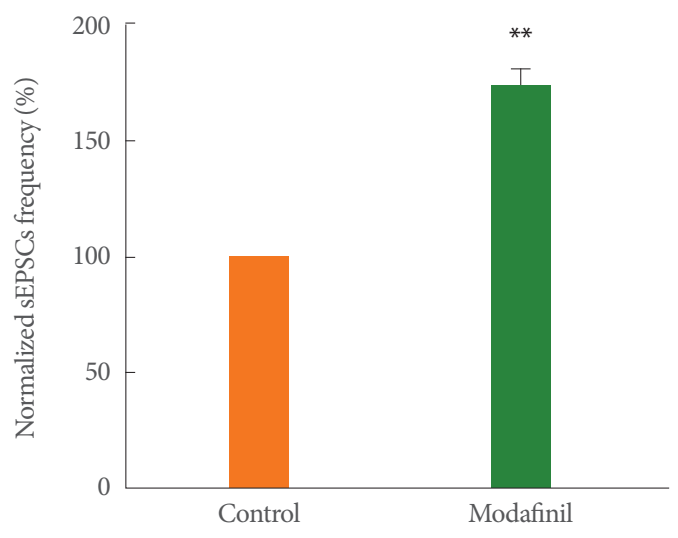

Modafinil

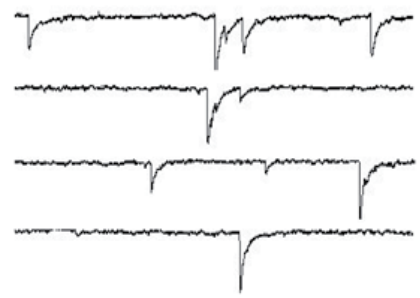

C
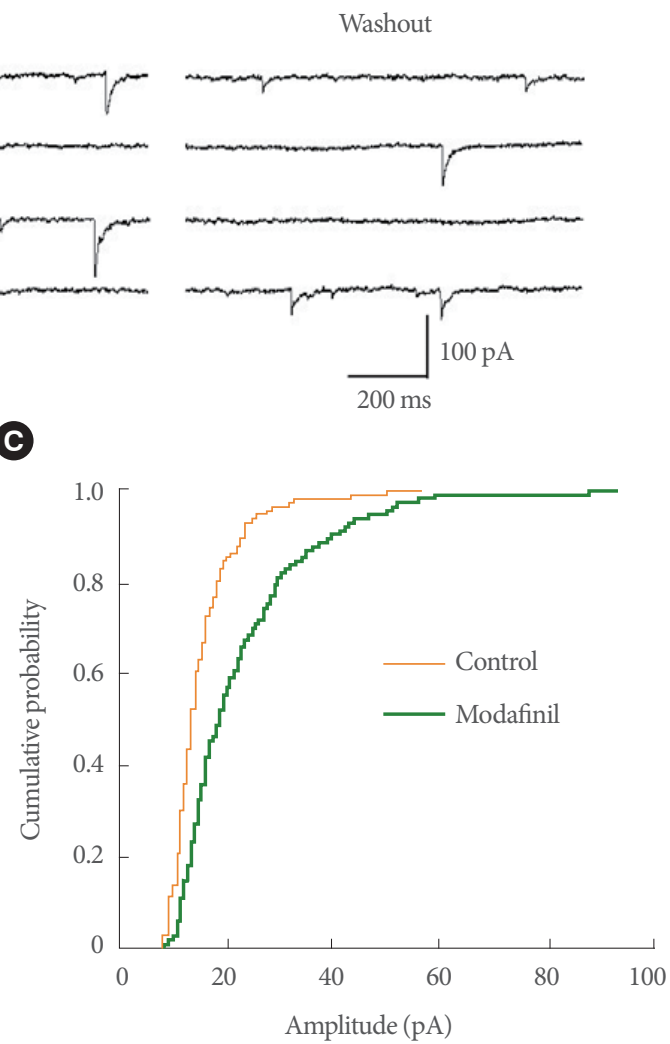

Fig. 4. Modafinil increased the frequency and amplitude of spontaneous excitatory postsynaptic currents (sEPSCs) in CA1 pyramidal neurons in hippocampal slices. (A) Sample traces represent sEPSCs recorded in a CA1 pyramidal neuron during $1 \mathrm{mM}$ modafinil treatment. (B) Modafinil increased the frequency of sEPSCs $(n=9, P<0.01)$. (C) Cumulative amplitude distribution of sEPSCs shows that modafinil increased the amplitude of sEPSCs $(n=9$, Kolmogorov-Smirnov test, $\mathrm{P}<0.01)$. Membrane holding potential, $-70 \mathrm{mV}$. ** $\mathrm{P}<0.01$ compared with the control group.

control, 8 slices, 5 animals; $\mathrm{P}<0.01$ ) (Fig. 5C).

\section{DISCUSSION}

Our studies demonstrated the effects of chronic i.p. modafinil administration on behavioral learning in healthy, non-sleepdeprived adult rats. We found that modafinil produced a dosedependent improvement in Y-maze learning performance, and the enhancement did not increase further when the daily dose was greater than $200 \mathrm{mg} / \mathrm{kg}$ per training day (Fig. 2). Our results are consistent with a previous study, which reported that daily administration of modafinil before training improved acquisition on a Morris water maze and increased visuo-spatial long-term memory performance $[10,11]$. Our data provide evidence for modafinil to be considered as a cognitive enhancer.

The mechanisms underlying the enhancement of learning and memory by modafinil are still not well understood. It is well known that information processing in the mammalian brain is governed by a dynamic interplay between excitatory and inhibitory neurotransmission [17]. Changes in excitatory synaptic transmission or inhibitory synaptic transmission may affect learning behavior. Enhancement of excitatory transmission in the hippocampal dentate gyrus facilitates Y-maze learning performance [12], while a reduction in excitatory transmission causes deficits in hippocampus-dependent spatial learning and memory [18]. However, enhanced inhibitory synaptic transmission in the dentate gyrus of Ts65Dn mice is implicated in impaired cognition during behavioral tasks mediated by the hippocampus [13]. Moreover, 2-deoxyglucose autoradiography studies have shown that modafinil substantially modifies the activity of all the subregions of the hippocampus (subiculum, CA1-CA3, and dentate gyrus) [19]. Modafinil has also been shown to reduce the release of extracellular GABA, which decreases GABAergic transmission [6], and enhance the gluta- 
A

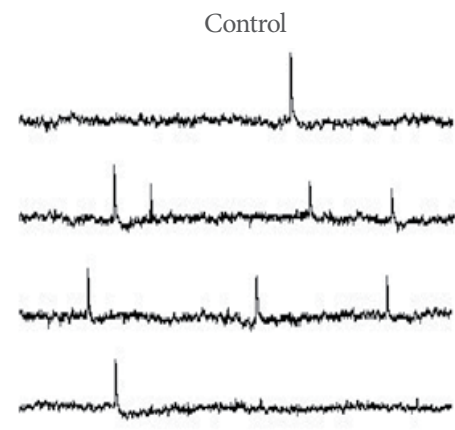

B

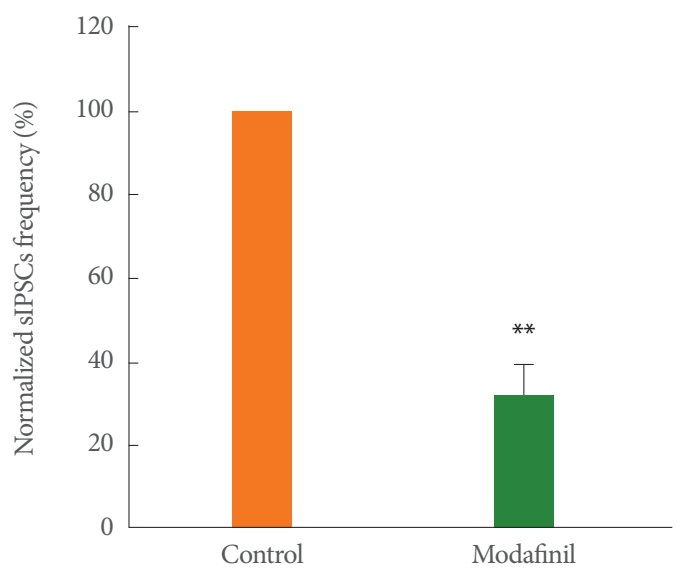

Modafinil

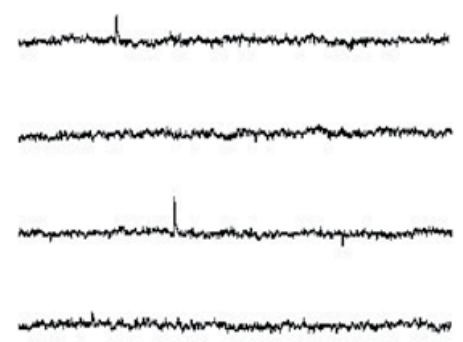

C

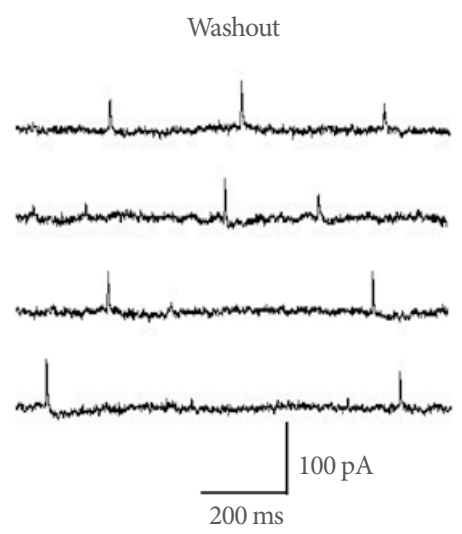

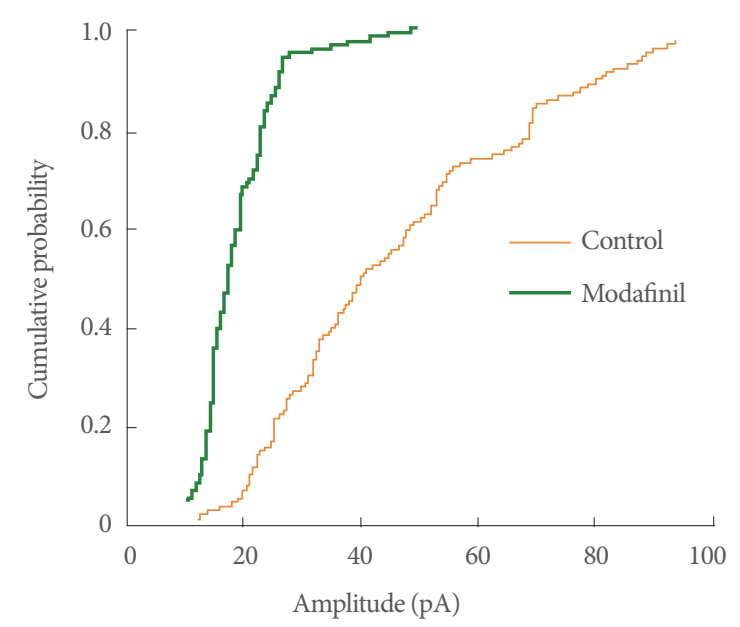

Fig. 5. Modafinil decreased the frequency and amplitude of spontaneous inhibitory postsynaptic currents (sIPSCs) in CA1 pyramidal neurons in hippocampal slices. (A) Sample traces represent sIPSCs recorded in a CA1 pyramidal neuron during $1 \mathrm{mM}$ modafinil treatment. (B) Modafinil decreased the frequency of sIPSCs $(n=8, P<0.01)$. (C) Cumulative amplitude distribution of sIPSCs shows that modafinil decreased the amplitude of sIPSCs $(n=8$, Kolmogorov-Smirnov test, $\mathrm{P}<0.01)$. Membrane holding potential, $-10 \mathrm{mV}$. ${ }^{* *} \mathrm{P}<0.01$ compared with the control group.

mate release associated with a lack of effect on GABA release in the hippocampus and thalamus [8]. Thus, it is plausible that chronic modafinil affected Y-maze learning performance by altering synaptic transmission in the hippocampus. To determine the effects of modafinil on synaptic transmission, sPSCs in pyramidal neurons of hippocampal CA1 slices were examined in the presence of modafinil. The frequency and amplitude of sPSCs were reversibly enhanced by modafinil (Fig. 3). As sPSCs represent the sum of glutamatergic sEPSCs and GABAergic sIPSCs, the effects of modafinil on sEPSCs and sIPSCs were also assessed. Our results showed that perfusion with modafinil enhanced the frequency and amplitude of sEPSCs in hippocampal slices (Fig. 4), suggesting that the enhancement of glutamate-mediated synaptic transmission by modafinil may be one of the mechanisms underlying its effect on Y-maze learning. We also found that the frequency and amplitude of sIPSCs were reduced by modafinil perfusion (Fig. 5). In the hippocampus, an increase in GABAergic inhibition is thought to attenuate the encoding of episodic behavioral memory [20]. GABAergic synaptic inhibition plays a critical role in regulating the induction of long-term potentiation (LTP), and the LTP of synapses is assumed to be one of the cellular mechanisms for learning and memory. Previous studies have shown that the blockade of GABAa receptor-mediated inhibition facilitates LTP induction [21,22], and this facilitated induction has been shown to contribute to improvements in Y-maze learning performance [12]. Therefore, our present study indicates that the inhibition of GABAergic synaptic transmission by modafinil 
may also contribute to improvements in the rats' Y-maze learning performance.

In conclusion, the present study demonstrates that chronic modafinil administration enhances Y-maze learning performance in healthy, non-sleep-deprived adult rats. The potential mechanisms at least partly include the enhancement of glutamatergic excitatory synaptic transmission and the inhibition of GABAergic synaptic transmission in the hippocampus. Our results have proved the role of modafinil as a cognitive enhancer.

\section{REFERENCES}

1. Didato G, Nobili L. Treatment of narcolepsy. Expert Rev Neurother 2009;9:897-910.

2. Smith D, Pernet A, Rosenthal JM, Bingham EM, Reid H, Macdonald IA, et al. The effect of modafinil on counter-regulatory and cognitive responses to hypoglycaemia. Diabetologia 2004;47:170411.

3. Kraft GH, Bowen J. Modafinil for fatigue in MS: a randomized placebo-controlled double-blind study. Neurology 2005;65:1995-7.

4. Nieves AV, Lang AE. Treatment of excessive daytime sleepiness in patients with Parkinson's disease with modafinil. Clin Neuropharmacol 2002;25:111-4.

5. Minzenberg MJ, Carter CS. Modafinil: a review of neurochemical actions and effects on cognition. Neuropsychopharmacology 2008;33:1477-502.

6. Pierard C, Liscia P, Philippin JN, Mons N, Lafon T, Chauveau F, et al. Modafinil restores memory performance and neural activity impaired by sleep deprivation in mice. Pharmacol Biochem Behav 2007;88:55-63.

7. Beracochea D, Cagnard B, Celerier A, le Merrer J, Peres M, Pierard C. First evidence of a delay-dependent working memory-enhancing effect of modafinil in mice. Neuroreport 2001;12:375-8.

8. Pierard C, Liscia P, Valleau M, Drouet I, Chauveau F, Huart B, et al. Modafinil-induced modulation of working memory and plasma corticosterone in chronically-stressed mice. Pharmacol Biochem Behav 2006;83:1-8.

9. Beracochea D, Celerier A, Borde N, Valleau M, Peres M, Pierard C. Improvement of learning processes following chronic systemic administration of modafinil in mice. Pharmacol Biochem Behav 2002;73:723-8.

10. Shuman T, Wood SC, Anagnostaras SG. Modafinil and memory: effects of modafinil on Morris water maze learning and Pavlovian fear conditioning. Behav Neurosci 2009;123:257-66.

11. Burgos H, Castillo A, Flores O, Puentes G, Morgan C, Gatica A, et al. Effect of modafinil on learning performance and neocortical long-term potentiation in rats. Brain Res Bull 2010;83:238-44.

12. Rao Y, Xiao P, Xu S. Effects of intrahippocampal aniracetam treatment on Y-maze avoidance learning performance and behavioral long-term potentiation in dentate gyrus in rat. Neurosci Lett 2001;298:183-6.

13. Kleschevnikov AM, Belichenko PV, Faizi M, Jacobs LF, Htun K, Shamloo $\mathrm{M}$, et al. Deficits in cognition and synaptic plasticity in a mouse model of Down syndrome ameliorated by GABAB receptor antagonists. J Neurosci 2012;32:9217-27.

14. Yao LH, Li CH, Yan WW, Huang JN, Liu WX, Xiao P. Cordycepin decreases activity of hippocampal CA1 pyramidal neuron through membrane hyperpolarization. Neurosci Lett 2011;503:256-60.

15. Zhou Y, Tang H, Liu J, Dong J, Xiong H. Chemokine CCL2 modulation of neuronal excitability and synaptic transmission in rat hippocampal slices. J Neurochem 2011;116:406-14.

16. Urbanski MJ, Kovacs FE, Szabo B. Endocannabinoid-mediated synaptically evoked suppression of GABAergic transmission in the cerebellar cortex. Neuroscience 2010;169:1268-78.

17. Martin LJ, Zurek AA, MacDonald JF, Roder JC, Jackson MF, Orser BA. Alpha5GABAA receptor activity sets the threshold for longterm potentiation and constrains hippocampus-dependent memory. J Neurosci 2010;30:5269-82.

18. Kouser M, Speed HE, Dewey CM, Reimers JM, Widman AJ, Gupta $\mathrm{N}$, et al. Loss of predominant Shank3 isoforms results in hippocampus-dependent impairments in behavior and synaptic transmission. J Neurosci 2013;33:18448-68.

19. Engber TM, Dennis SA, Jones BE, Miller MS, Contreras PC. Brain regional substrates for the actions of the novel wake-promoting agent modafinil in the rat: comparison with amphetamine. Neuroscience 1998;87:905-11.

20. Steckler T, Sahgal A, Aggleton JP, Drinkenburg WH. Recognition memory in rats--III. Neurochemical substrates. Prog Neurobiol 1998;54:333-48.

21. Meredith RM, Floyer-Lea AM, Paulsen O. Maturation of long-term potentiation induction rules in rodent hippocampus: role of GABAergic inhibition. J Neurosci 2003;23:11142-6.

22. Wigstrom H, Gustafsson B. Facilitated induction of hippocampal long-lasting potentiation during blockade of inhibition. Nature 1983;301:603-4. 